\title{
Die pertrochantere Femurfraktur
}

\author{
Rolle der Endoprothetik
}

\author{
Eugen Winter, Kuno Weise
}

\section{Zusammenfassung}

Der überwiegende Teil der proximalen Femurfrakturen Typ 31 A 1 -3 ist zuverlässig mittels Osteosynthese zur Ausheilung zu bringen. In folgenden Ausnahmefällen kommt eine operative Versorgung mit einem künstlichen Hüftgelenk bei diesen Frakturtypen in Betracht: vorbestehende versteifende Hüftarthrose, kombinierte peritrochantere Femurfrakturen beim alten Menschen, pathologische 31 A1 -3-Frakturen, Kombination einer Azetabulumfraktur mit einer proximalen Femurfraktur beim alten Menschen und bei bestimmten Komplikationen nach einer Osteosynthese einer proximalen Femurfraktur. Der Anteil dieser besonderen Situationen wird mit zunehmendem Anteil alter Menschen an der Bevölkerung steigen.

\section{Einleitung}

Die konservative Behandlung proximaler Femurfrakturen Typ 31 A 1 - 3 [4] (Abb.1) ist heute so gut wie nicht mehr angezeigt. Ein langwieriger Streck- oder Gipsverband würde alle Nachteile der Bettlägerigkeit wie Pneumonie, Thrombose, Embolie oder Dekubitus mit sich bringen. Stellvertretend für eine Vielzahl von Publikationen, die den großen Erfolg der Osteosynthese der pertrochanteren Frakturen dokumentieren, seien hier folgende Resultate der AO-Arbeitsgruppe „Osteosynthesen am proximalen Femur" aufgeführt [3]: In der BG-Unfallklinik Tübingen

OP-JOURNAL 2002; 17: 132-140

(c) Georg Thieme Verlag Stuttgart · New York und zehn weiteren Kliniken wurden im Jahr 1996 insgesamt 320 Patienten erfasst, die wegen einer 31 A 2 oder 31 A 3-Fraktur (Abb.1) des proximalen Femurs behandelt wurden. Das Durchschnittsalter lag bei 83 Jahren, das Verhältnis Frauen : Männer betrug 4,9 : 1. In 171 Fällen wurde eine DHS, in 90 Fällen ein PFN, bei 44 Patienten ein Gamma-Nagel, bei 8 Patienten eine Kondylenplatte und in 7 Fällen eine DCS verwendet. In keinem Fall wurde beim primären Eingriff eine Endoprothese implantiert. In lediglich 8 Fällen (2,5\%) wurde eine Reoperation erforderlich: 6-mal eine Reosteosynthese (4-mal PFN, 1-mal Gammanagel, 1-mal Kondylenplatte), 1-mal wurde eine Resektionsarthroplastik (Girdlestone-Situation) notwendig und in nur einem Fall $(0,3 \%)$ wurde die Indikation zur Implantation einer Hüftendoprothese gesehen. In der Studie waren nicht die 31 A 1-Frakturen (Abb.1) berücksichtigt, die noch am ehesten die Implantation eines Kunstgelenkes erlauben, sofern bestimmte Begleitumstände vorliegen, die nachfolgend ausführlich dargestellt werden.

Es muss deutlich hervorgehoben werden, dass die pertrochanteren Frakturen nur in sehr seltenen Ausnahmefällen eine Indikation für einen endoprothetischen Eingriff darstellen.

Die pertrochanteren Femurfrakturen Typ $31 \mathrm{~A} 1$ und $31 \mathrm{~A} 2$ sind meistens sehr gut mittels Osteosynthese $\left(\mathrm{DHS}^{\circledR}, \mathrm{PFN}^{\circledR}\right.$,

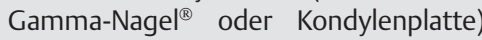
zur Ausheilung zu bringen. Eine Indikation zur primären Implantation einer Hüftendoprothese besteht nur in seltenen Ausnahmefällen oder bei bestimmten Komplikationen nach primärer Osteosynthese dieser Frakturen.

\section{Hauptteil}

Wegen der sehr guten Resultate der Osteosynthesen bei den pertrochanteren Frakturen sollte man immer versuchen, die originäre Knochenanatomie wiederherzustellen, es sei denn, es liegen die an- gesprochenen speziellen Begleitumstände vor, auf die im Weiteren eingegangen wird. Zudem ist es für eine erfolgreiche Hüftendoprothesenimplantation von größter Bedeutung, dass das intertrochantere Areal intakt ist, da bei den meisten modernen Schaftprothesen gerade dieser Bereich die wichtigste Verankerungszone des Implantates darstellt [5]. Die Intaktheit des Trochanter major ist auch für die muskuläre Stabilisierung des Hüftgelenkes außerordentlich wichtig. Am Trochanter major inserieren wichtige Hüftmuskeln. An seiner Spitze setzt die Sehne des M. piriformis an, an seiner Hinter- und Seitenfläche der M. gluteus medius, ventrolateral der M. gluteus minimus und in der Fossa trochanterica, an der medialen Fläche des Trochanter major, die kurzen Außenrotatoren (Mm. gemellus superius, obturatorius internus, gemellus inferius und obturatorius externus). Die vom Trochanter major nach kranial ziehenden Muskel- und Sehnenanteile wirken als laterale Zuggurtung und stellen wesentliche Stabilisatoren für das Hüftgelenk dar. Geht diese stabilisierende Zuggurtungswirkung durch eine Dislokation des Trochanter major verloren, führt dies zu einem Trendelenburgschen Hinken. Der Trochanter minor ist der Insertionspunkt für die kräftige Sehne des M. iliopsoas und bildet einen Teil der massiven medialen Abstützung des inneren Schenkelhalsrandes. Es spricht demnach vieles dafür, dass dieser Bereich im Falle einer knöchernen Verletzung wieder bestmöglich durch eine Osteosynthese wiederhergestellt wird.

Der intakte intertrochantere Bereich ist für die muskuläre Verspannung des Hüftgelenkes von größter Bedeutung, da am Trochanter major und minor wichtige Stabilisatoren ansetzen. Die Intaktheit dieses Areals ist auch deshalb wichtig, da die meisten modernen Endoprothesenschäfte ihre Hauptverankerung intertrochanter haben. Die bestmögliche Rekonstruktion dieses Bereiches ist von elementarer Bedeutung. 


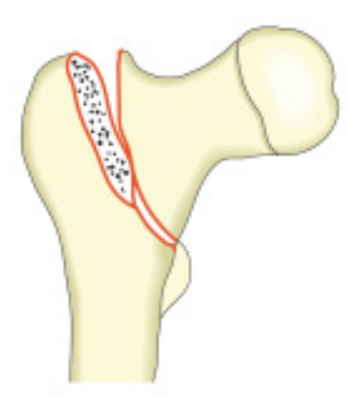

A 1.1

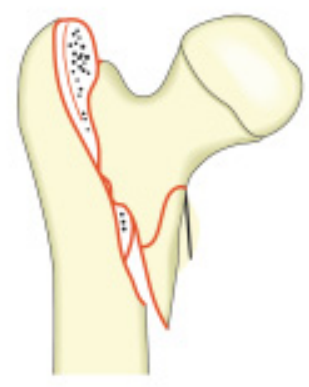

A 2.1

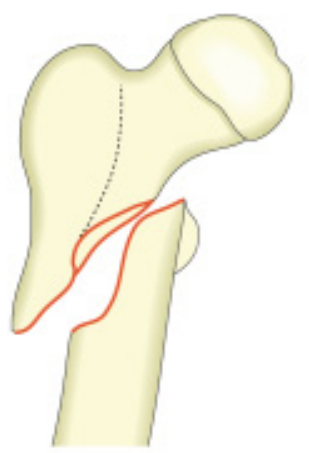

A 3.1

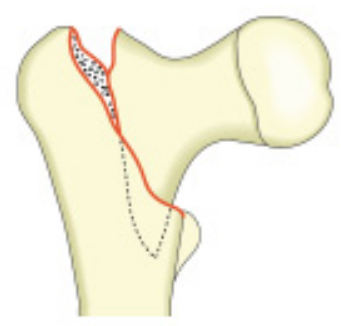

A 1.2

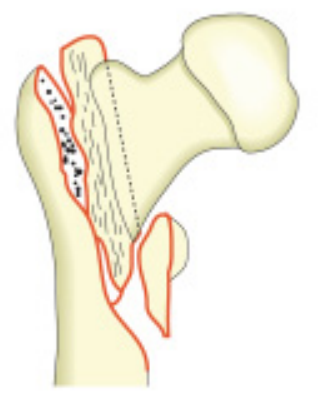

A 2.2

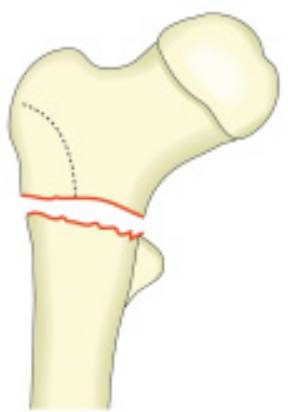

A 3.2

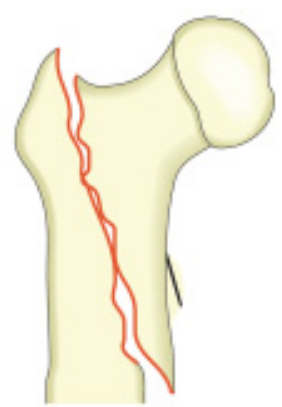

A 1.3

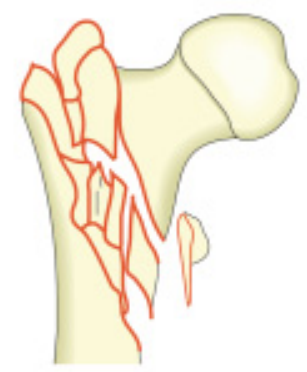

A 2.3

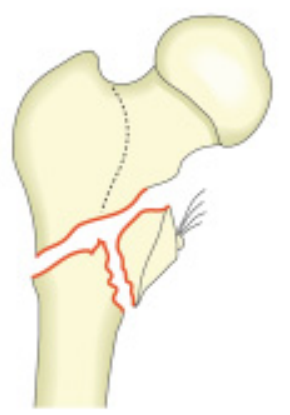

A 3.3

Abb.1 Einteilung der Frakturen im Bereich der Trochanterregion (nach Müller et al [4]).

Es gibt jedoch eine Reihe von Ausnahmen, die den Chirurgen dazu veranlassen, nach einer Alternative zur Osteosynthese zu suchen:

\section{Vorbestehende versteifende Hüftarthrose bei 31 A 1, A2 oder A3-Frakturen}

Liegt bei einer dieser Frakturen am coxalen Femurende eine weit fortgeschrittene, einsteifende Arthrose vor, so müssen folgende zwei Überlegungen angestellt werden: A) Die Osteosynthese einer hüftgelenksnahen Fraktur kann durch die fast aufgehobene Beweglichkeit des betroffenen Hüftgelenkes massiv erhöhten biomechanischen Belastungen ausgesetzt sein. Ist die Knochenqualität wie bei den meist alten Patienten reduziert, erhöht sich die Gefahr nochmals, dass das
Implantat den Belastungen nicht standhält. B) Hatte der Patient schon vor dem Erleiden der Fraktur starke Schmerzen seitens einer einsteifenden Hüftarthrose, so ist die gleichzeitige Therapie dieser vorbestehenden Coxarthrose mit ins Kalkül zu ziehen. Im Text wird noch dargestellt werden, dass man sich bei den 31 A 1-Frakturen eher für eine Endoprothese entscheiden kann, weniger leicht bei den 31 A2-Frakturen und nur mit großem Vorbehalt bei den 31 A3Frakturen. Würde man in diesen Fällen zunächst die Osteosynthese und danach als einen weiteren Eingriff den Einbau eines Kunstgelenkes erwägen, würde man die meist alten Patienten, welche auch häufig an schwerwiegenden Nebenerkrankungen leiden, zwei Mal einem Operationsrisiko aussetzen.
Pathologische 31 A1, A2 oder A3-Frakturen

Liegt eine pathologischen Fraktur in dieser anatomischen Region vor, so sind ergänzende Überlegungen anzustellen. Bekanntlich kann es sich bei einer pathologischen Fraktur ursächlich sowohl um einen benignen als auch um einen malignen Prozess handeln. Auch eine Fraktur bei einer fortgeschrittenen Osteoporose ist als pathologische Fraktur zu bezeichnen. Liegt bei einem jüngeren Patienten eine gutartige statikschwächende Knochenzyste im intertrochanteren Bereich vor, so wird man sicherlich eine hüftkopferhaltende Osteosynthese zusammen mit einer Knochentransplantation erwägen. Handelt es sich jedoch um eine Metastase eines Malignoms (z.B. eines Mammaoder Prostatakarzinoms), so sind wiederum zwei Überlegungen anzustellen:

A) Möglicherweise ist eine Verbundosteosynthese mit einer $95^{\circ}$-Kondylenplatte unter Verwendung von Knochenzement zum Auffüllen des durch den Tumor bedingten Knochendefektes sinnvoll. B) Ist der Ausgang einer solchen Verbundosteosynthese durch die individuellen Gegebenheiten fraglich, so ist die Implantation einer Endoprothese in Betracht zu ziehen. Dabei ist das Ausmaß der knöchernen Zerstörung im intertrochanteren Bereich des Femurs zu berücksichtigen. Je nachdem wird man sich bei den weniger ausgedehnten Defekten mit gut erhaltenem Femurrohr für die Implantation einer zementierten Standardprothese oder für eine ausgedehntere Knochenresektion entscheiden, was dann aber die Implantation einer ,Tumorprothese“ erforderlich macht (Abb.2 u.9).

\section{Kombinierte peritrochantere Frakturen} beim alten Menschen

Es kann zu einer Kombination verschiedener Frakturen um den Bereich des Trochantermassives (peritrochantere Fraktur) kommen, z.B. kann eine mediale Schenkelhalsfraktur mit einer subtrochanteren Fraktur kombiniert sein. Beim alten Menschen muss in einem solchen Falle überlegt werden, ob nicht eine definitive Versorgung dieser Fraktur mit einer Endoprothese ratsam ist, da auf diese Weise ein eventueller Zweiteingriff bei potentiell möglicher Hüftkopfnekrose vermieden werden kann. Bei einem jungen Patienten wird man jede Anstrengung unternehmen, den Hüftkopf zu erhalten. Einem jüngeren Patienten kann eher eine möglicherweise notwendige 


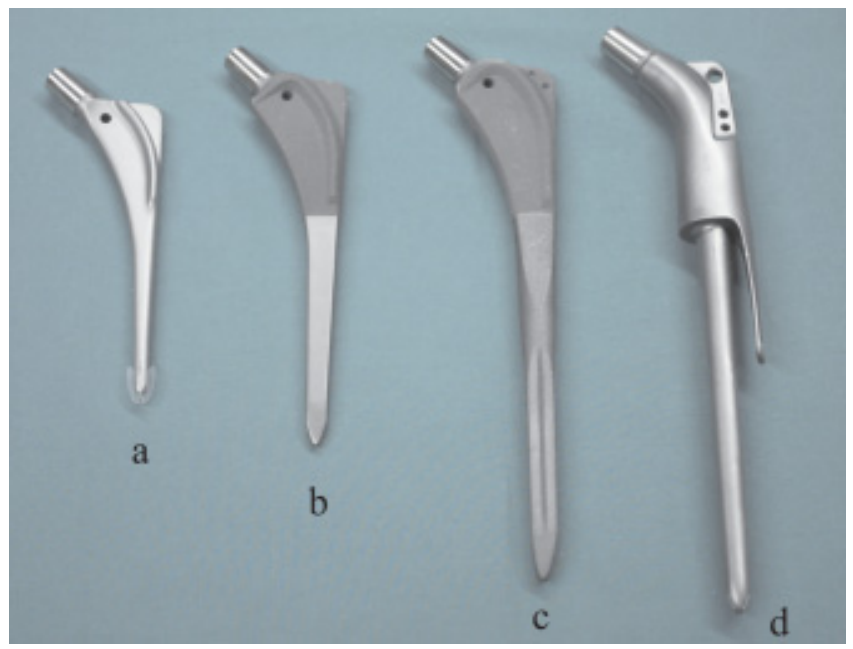

Abb. 2 (a) zementierte unbeschichtete BiContact ${ }^{\mathbb{B}}$ Schaftprothese mit Centralizer, (b) mit Plasmopore ${ }^{\circledR}$ beschichteter BiContact ${ }^{\circledR}$-Schaft zur zementfreien Verankerung, (c) BiContactRevisionsschaft ${ }^{\circledR}$ und (d) Tumorprothese ${ }^{\circledR}$ (alle Implantate Fa. Aesculap, Tuttlingen).

Zweitoperation im Falle einer Hüftkopfnekrose zugemutet werden. Die Entscheidung ist in jedem Fall individuell und hängt sehr von der Frakturkombination und den Begleitumständen jedes einzelnen Patienten ab.

Begleitende Azetabulumfraktur beim alten Patienten bei 31 A1, A2 oder A3-Frakturen oder knöcherne Metastasen im Azetabulum

Liegt bei einem alten Patienten zusätzlich eine operationsbedürftige azetabuläre Fraktur vor, so muss erwogen werden, ob nicht statt einer aufwändigen, belastenden Azetabulumosteosynthese die Implantation eines azetabulären Metallabstützringes [8] durchgeführt werden soll. In diesem Falle müsste aber auch eine Femurschaftprothese implantiert werden. Gut vorstellbar ist ein solches Vorgehen bei einem alten Patienten mit der Kombination einer 31 A 1 oder 31 A 2 Fraktur bei zusätzlicher operationswürdiger Azetabulumfraktur. Eine weitere Indikation für die Verwendung eines solchen Abstützringes kann das Vorliegen von Knochenmetastasen im Azetabulum sein.

\section{Komplikationen nach Osteosynthese eine}

\section{A1, A 2 oder A3-Fraktur}

Bei extrakapsulär gelegenen pertrochanteren Femurfrakturen ist die Gefahr der Verletzung der vulnerablen Blutversorgung des Hüftkopfes praktisch nicht gegeben, die laterale Epiphysenarterie aus der A. circumflexa femoris medialis als entscheidende Lebensader für den Hüftkopf wird bei den pertrochanteren Femurfrakturen nur selten mitverletzt, Verletzungen bei intramedullären Femursta- bilisierungen wie z.B. nach Versorgung einer solchen Fraktur mittels Gammanagel ${ }^{\circledR}$ oder proximalem Femurnagel $\left(\mathrm{PFN}^{\circledR}\right)$ jedoch beschrieben. Es kann auch zum Versagen einer Osteosynthese kommen, wenn beispielsweise das Implantat den biomechanischen Anforderungen der vorliegenden Fraktur nicht gerecht wird oder wenn es im Falle einer DHS ${ }^{\circledR}$, eines Gammanagels $^{\circledR}$ oder eines PFN ${ }^{\circledR}$ zum Auswandern der Schenkelhalsschrauben („cutting out“) kommt (Abb.7). Da aber in einem weiteren Beitrag auf derartige Komplikationen eingegangen wird, soll dies hier nur angedeutet aufgeführt werden.
Folgende Situationen können ausnahmsweise eine Indikation für eine primäre Hüftendoprothese bei den 31 A 1, A 2 und $A$ 3-Frakturen sein: Eine vorbestehende, einsteifende Hüftarthrose, pathologische pertrochantere Femurfrakturen, kombinierte peritrochantere Frakturen beim alten Menschen, begleitende Azetabulumfrakuren beim alten Menschen, bestehende knöcherne Metastasen im Azetabulum und Komplikationen nach Osteosynthese pertrochanterer Frakturen.

\section{Vorgehensweise bei der Endoprothesenimplantation in Bezug auf den Frakturtyp}

Die Gruppe 31 A 1 umfasst die einfachen pertrochanteren Frakturen (Abb.1). Bei der A 1.1 Fraktur und bei der A 1.2 Fraktur ist das intertrochantere Massiv mit Trochanter major und minor intakt. Somit ist bei diesen Frakturtypen eine wichtige biomechanische Voraussetzung für eine zuverlässige Verankerung eines primären Hüftendoprothesenschaftes gegeben. Schwieriger wird diese Verankerung dann schon bei der A 1.3-Fraktur, da hier ein Verlust der wichtigen medialen Abstützung, des Trochanter minor-Bereiches besteht.

Liegt bei Patienten mit solchen Frakturen eine schon weit fortgeschrittene, versteifende Coxarthrose vor oder handelt es sich um eine pathologische Fraktur bei

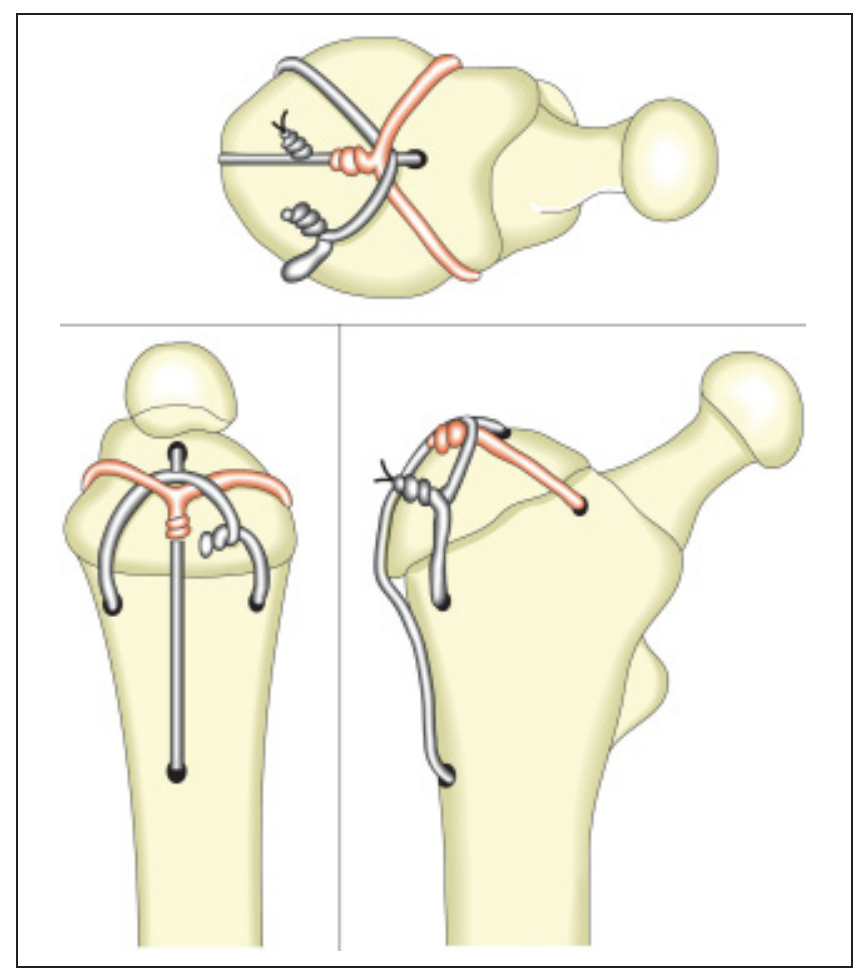

Abb. 3 Von Charnley [1] angegebenes gekreuztes Drahtcerclagensystem mit doppeltem vertikalen Draht (schwarze Linie). 
ausgeprägter Osteoporose oder malignem Grundleiden, so kann eine endoprothetische Versorgung indiziert sein. Hat man sich zu einer solchen entschlossen, dann kann dieser Eingriff auch geplant durchgeführt werden. Eine dringliche Operationsindikation ist deshalb nicht mehr gegeben, da es nicht mehr um den Kopferhalt geht, der ein rasches operatives Einschreiten erfordert. So kann bei einem relativ schmerzbefreiten $\mathrm{Pa}$ tienten mit pertrochanterer Fraktur und der Indikation $\mathrm{zu}$ einer Endoprothese eine Wartezeit akzeptiert werden, in der diese meist alten und zusätzlich erkrankten Patienten anästhesiologisch gut vorbereitet werden können.

Bei entsprechender Indikation ist bei den 31 A 1.1. und 31 A 1.2 Frakturen eine Hüftendoprothesenimplantation technisch relativ einfach und sicher durchzuführen. Bei allen anderen 31 A-Frakturtypen ist die Implantation dadurch erschwert, dass die zuverlässige Refixation des Trochanterbereiches sehr schwierig sein kann.

Bei den 31 A 1.1 und 31 A 1.2-Frakturen ist bei guter Knochenqualität theoretisch eine zementfreie Schaftimplantation [5] denkbar. Der Chirurg wird sich jedoch vorzugsweise für eine zementierte Verankerung [5] des Hüftendoprothesenschaftes entschließen, weil a) die optimale metaphysäre Verankerung des Schaftes durch eine mögliche zusätzliche, möglicherweise nicht auf dem Röntgenbild sichtbare Schaftfissur bei den A 1.1 und A 1.2 Frakturen beeinträchtigt sein kann, b) die Knochenqualität bei Osteoporose oder ossärer Metastase erheblich reduziert sein kann und c) bei den zumeist alten Patienten postoperativ eine rasche Mobilisierung mit voller Belastung des verletzen Beines wünschenswert und bei Zementierung auch möglich ist. Aus eigener Erfahrung raten wir bei diesen Frakturen von einer zementfreien Schaftverankerung ab, da die Gefahr des Einsinterns des Endoprothesenschaftes viel zu groß ist.

Besteht die Indikation zur endoprothetischen Versorgung, so kann diese in Form einer Teilprothese (Kopfersatzprothese) oder als Hüftgelenktotalendoprothese erfolgen. Bei der Teilprothese werden die Großkopfprothese und das Doppelkopfsystem unterschieden. Im Falle der Großkopfprothese wird das Kopfersatzteil entsprechend der Azetabulumgröße auf den Schaftkonus aufgesetzt. Die Indikation ist sehr streng zu stellen, da es bei längerer Liegedauer zum Durchwandern des Kopfes durch den nicht ersetzten Pfannenboden kommen kann. Das Doppelkopfsystem (Duokopfprothese, bipolare Kopfprothese) beruht auf dem Prinzip des Pfannenersatzes ohne mechanische Verankerung desselben im Azetabulum. Der Prothesenkopf rotiert in einem Inlay aus Polyethylen (PE), welches fest mit einer Metallschale verbunden ist. Der Durchmesser dieser Metallschale entspricht der Azetabulumgröße, welche intraoperativ bestimmt werden kann. Dieses System kann noch zusätzlich im Azetabulum rotieren. In Deutschland hat die Duokopfprothese bei der Frakturversorgung mittlerweile eine weite Verbreitung gefunden. Wir sind in unserer Klinik mit der Indikation zur Duokopfprothese eher zurückhaltend, da wir auch bei diesem System schon beträchtliche Durchwanderungen durch den Beckenboden gesehen haben.

Der komplette Hüftgelenkersatz mit zementierter PE-Pfanne und zementiertem Schaft kann bei dieser Indikation als der „Goldstandard“ angesehen werden. Es gibt zwar eine Vielzahl verschiedener zementierte Endoprothesenmodelle am Hüftgelenk, die Ansichten über die optimale operative Technik haben sich in den letzen Jahren jedoch weitestgehend vereinheitlicht. Nachfolgend soll die in unserer Klinik gebräuchliche Vorgehensweise dargestellt werden: Der Pat. wird auf dem Normaltisch auf dem Rücken gelagert. Auf eine neutrale Stellung des Tisches muss präoperativ dringend geachtet werden, da es bei gekipptem Tisch leicht zu einer Fehlpositionierung der Implantate kommen kann. Es erfolgt die dreimalige Hautdesinfektion des gesamten verletzen Beines und die sterile Abdeckung mit Einwegmaterial. Eine Inzisionsfolie wird nicht mehr verwendet. An der proximalen Oberschenkelaußenseite wird die Haut auf einer Strecke von $20 \mathrm{~cm}$ inzidiert $(10 \mathrm{~cm}$ oberhalb und $10 \mathrm{~cm}$ unterhalb des Trochanter major). Nach Durchtrennen des Subkutangewebes und der Oberschenkelfaszie wird der M. gluteus medius auf einer Strecke von etwa $3-5 \mathrm{~cm}$ von dessen Ansatz am Trochanter major abgelöst. Mit Hohmann-Hebeln werden der Schenkelhals und der vordere Azetabulumrand eingestellt. Dann wird die ventrale Hüftgelenkskapsel t-förmig inzidiert und reseziert. Mit der oszillierenden Säge erfolgt die laterale Schenkelhalsosteotomie, das Kopf-Hals-Fragment wird entfernt. Jetzt kann der hintere Pfannenrand mit Hohmann-Hebeln eingestellt werden. Das
Azetabulum muss sorgfältig freipräpariert werden. Mit den Kopffräsen wird beginnend bei $44 \mathrm{~mm}$ das Azetabulum aufgefräst, bis die spongiöse Struktur des Pfannenbodens „angeknackt“ ist, was sich in Form von vielen kleinen Blutpunkten zeigt. Dieser so vorbereitete Pfannenboden ist eine wichtige Voraussetzung sowohl für die zementfreie als auch für die zementierte Verankerung der azetabulären Komponente. Sofern die Knochenqualität gut ist, kann der Einbau einer Press-Fit-Pfanne erwogen werden. Da dies bei den Frakturen älterer Menschen häufig nicht der Fall ist, wird auf diese Technik hier nicht näher eingegangen. Der sichere Weg bei reduzierter Knochenqualität ist ohne Zweifel die Verankerung einer PE-Pfanne mit Polymethylmethacrylat (PMMA = Knochenzement). Es wird eine Pfanne mit $2 \mathrm{~mm}$ geringerem Durchmesser als der der letzten Fräse gewählt. Als Innendurchmesser der Pfanne verwenden wir bei Frakturen $32 \mathrm{~mm}$. Bekanntermaßen ist die Luxationsgefahr des Kunstgelenkes bei Frakturen im Vergleich zur Implantation bei Hüftarthrose erhöht. Die Gefahr einer Luxation eines Kunstgelenkes ist unserer Ansicht nach mit dem 32-mm-Kopf geringer als mit dem 28-mm-Kopf. Nach Bohren von 2-3 Verankerungslöchern wird der Pfannengrund von Debris und Blut sorgfältig gereinigt. Am besten gelingt dies mit der Jetlavage, die sich später auch für die Schaftvorbereitung empfiehlt. Der Knochenzement wird mit frischen Handschuhen eingebracht und sorgfältig in die Spongiosa „einmassiert“, damit der Zement so weit wie möglich $(2-3 \mathrm{~mm})$ in die Spongiosastruktur eindringen kann. Je besser das Eindringen des Zementes (Intrusion) gelingt, umso günstiger ist die Prognose für eine langfristete Haltbarkeit. Dann wird die Pfanne in 45 Grad Inklination und 15 Grad Anteversion eingebracht, überschüssiger Zement wird sorgfältig entfernt und die Pfanne mit einem Applikator bis zum Aushärten des Zementes unter Kompression gebracht, währenddessen wird mit Spülung gekühlt. Vor Anwendung des Zementes teilen wir dies rechtzeitig dem Anästhesisten mit, damit dieser $\mathrm{H} 1$ und H2-Rezeptoren-Blocker intravenös verabreichen kann, um einer möglichen Reaktion auf das PMMA vorzubeugen. Auf die eventuell notwendige Implantation eines metallischen Abstützrings im Bereich des Azetabulums wurde schon hingewiesen, die Technik wurde in einem früheren OP-Journal-Beitrag beschrieben [8]. 


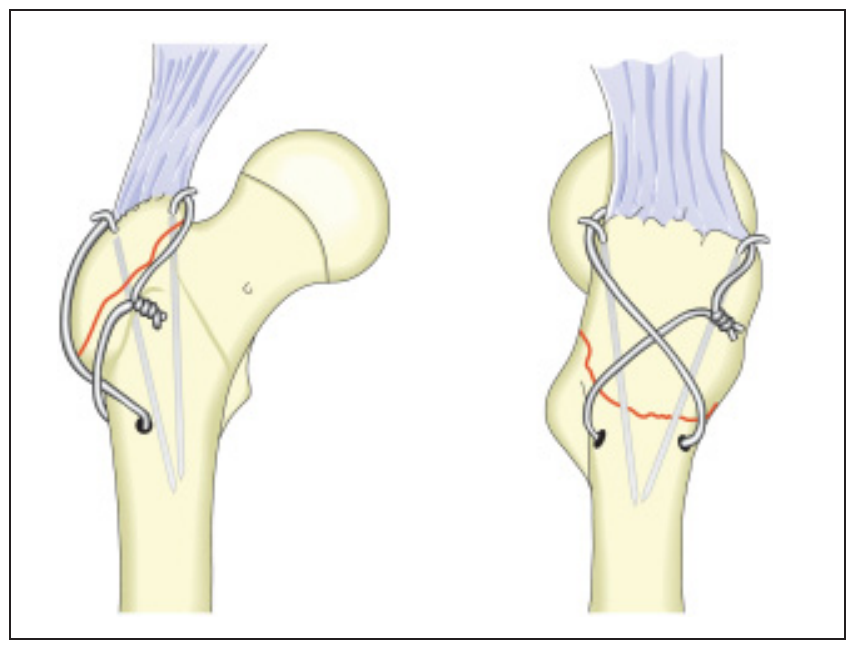

Abb. 4 Trochanterzuggurtung Einbringen von 2 K-Drähten durch den Trochanter in den Femurschaft. Durch ein queres Bohrloch im Femur wird ein Draht gezogen und als Achterschlinge um Sehnenansätze und K-Drähte geführt.
Das coxale Femurende wird unter Zug, Außenrotation und Adduktion eingestellt. In der BG-Klinik Tübingen wird seit nunmehr 15 Jahren der BiContact ${ }^{\circledR}$ Schaft (Abb.2) der Firma Aesculap mit großem Erfolg verwendet [5]. Es handelt sich um einen flachen Hüftendoprothesengeradschaft mit sehr hoher primärer Rotationsstabilität, der seine Verankerung in der Femurmetaphyse im Massiv zwischen dem Trochanter major und minor findet. Der Schaft wird mit so genannten A- und B-Profilern vorbereitet, dabei wird kein Knochen „weggeraspelt“ und damit geopfert, vielmehr wird der Knochen erhalten und verdichtet. Bei guter Knochenqualität kann entsprechend der letzten Profilergröße ein Schaft zementfrei im proximalen Femur verankert werden. Durch Press-fit und Tragrippen besteht eine sehr hohe primäre Stabilität. Der zementfreie Schaft ist mit einer mikroporösen Titanstruktur (Plasmapore ${ }^{\circledR}$ ) beschichtet, in welche Knochen nachge- wiesenermaßen einwächst, wodurch eine langfristige Sekundärstabilität erreicht wird. Wie schon aufgeführt, ist aber bei den zumeist alten Patienten mit pertrochanteren Frakturen die Knochenqualität für eine zementfreie Verankerung nicht ausreichend gut, so dass sich hier meistens das Zementieren des Schaftes empfiehlt (Abb.5). Dabei sind einige wichtige Punkte $\mathrm{zu}$ beachten. Nachdem das Schaftlager mit den Profilern vorbereitet ist, wird eine Markraumsperre so eingebracht, dass diese etwa $1 \mathrm{~cm}$ unterhalb der Prothesenspitze $\mathrm{zu}$ liegen kommt. Die Markraumsperre kann aus autogenem Knochen aus dem resezierten Hüftkopf gewonnen werden. Dies führen wir meistens nicht durch, da der Hüftkopf bei guter Qualität für die Knochenbank verwendet wird und bei Verdacht auf malignen Prozess im Hüftkopf (meistens ossäre Metastase) sollte dieses suspekte Knochenmaterial nicht anderswo hin verpflanzt werden.
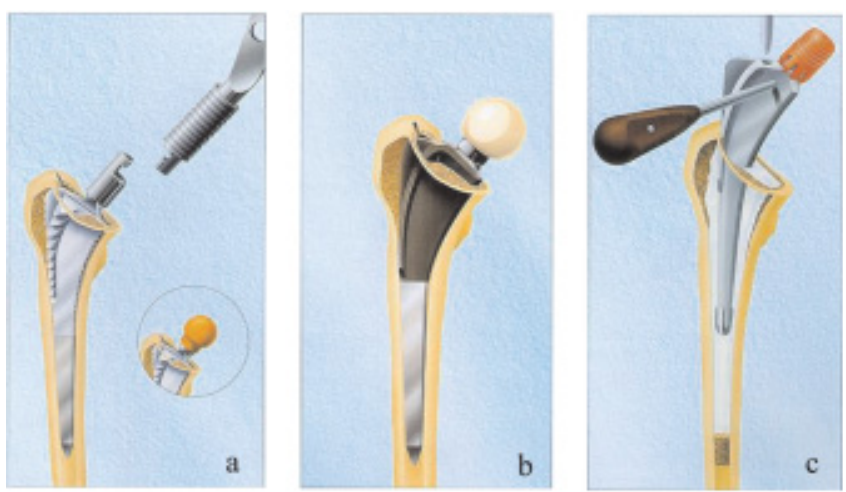

Abb.5 (a) Vorbereitung des Schaftlagers mit dem B-Profiler (Knochen wird erhalten und verdichtet, mit B-Profiler ist Probereposition möglich), (b) im Schaftlager kann ein Bi-Contact ${ }^{\circledR}$-Schaft zementfrei oder (c) zementiert (mit Centralizer und Markraumsperre) verankert werden.

In einem nächsten Schritt wird das Schaftlager mit der Jetlavage gereinigt. Bei den 31 A 1.1 -A 1.3 Frakturen muss der Trochanter nicht refixiert werden. Dies spielt bei den Frakturen an 31 A.2Fraktur eine wichtige Rolle und wird deshalb dort detailliert abgehandelt. In das so vorbereitete Schaftlager wird nun der Knochenzement eingebracht. In unserer Klinik geschieht dies mit der Zementpistole, der von Hand angerührte Zement wird damit von retrograd in das Schaftrohr eingebracht. Zunehmend mehr wird in Deutschland auch die Vakuumzementiertechnik verwendet. Der unbeschichtete BiContact ${ }^{\circledR}$-Schaft wird nun in anatomisch korrekter Position (bei Geradschaft in der neutralen Schaftachse mit 10 Grad Anteversion) in das Knochenrohr eingeführt. Für eine optimale Zentrierung wird auf die Schaftspitze ein Centralizer aus Kunststoff aufgesteckt. Am Schafteintritt wird durch ein entsprechendes Plastikteil oder durch Kompressen ein Gegendruck aufgebaut. Während der Zement aushärtet, wird der Schaft mit dem Applikator unter Druck gehalten und mit Spülung gekühlt. Ziel dieser Maßnahmen (Markraumsperre, Jetlavage, Zementkompression) ist es, eine bestmögliche Intrusion des Zementes (2$3 \mathrm{~mm}$ ) in die trabekuläre Knochenstruktur zu erreichen, was die Haltbarkeit des Kunstgelenkes verbessern hilft. Schließlich wird auf den Konus des Schaftes ein Probekopf aufgesteckt, das Kunstgelenk wird reponiert, es werden die Weichteilspannung und in den entsprechenden Provokationsstellungen des Hüftgelenkes die Luxationsneigung getestet. Dann wird der entgültige Stahl- oder Keramikkopf aufgesteckt, das Gelenk wird definitiv reponiert und die Tests werden nochmals durchgeführt. Es erfolgt der schichtweise Wundverschluss mit Drainageneinlage. Wenn keine maligne Erkrankung vorliegt, so wird ein Blutrückgewinnungssystem verwendet. Noch in Narkose erfolgt eine Röntgenaufnahme der operierten Hüfte in 2 Ebenen. Mit zementierter Pfanne und zementiertem Schaft versorgt kann der Patient ab dem zweiten postoperativen Tag aufstehen und voll belasten, wenn nicht eine Trochanterrefixierung (dann 1 Woche Bettruhe) erforderlich wurde.

Die 31 A 2 und 31 A 3 (Abb.1) umfasst die mehrfragmentären Frakturen dieser Region.

Hier ist die Implantation einer primären zementfreien, proximal verankerten Schaftprothese auf keinen Fall mehr mög- 

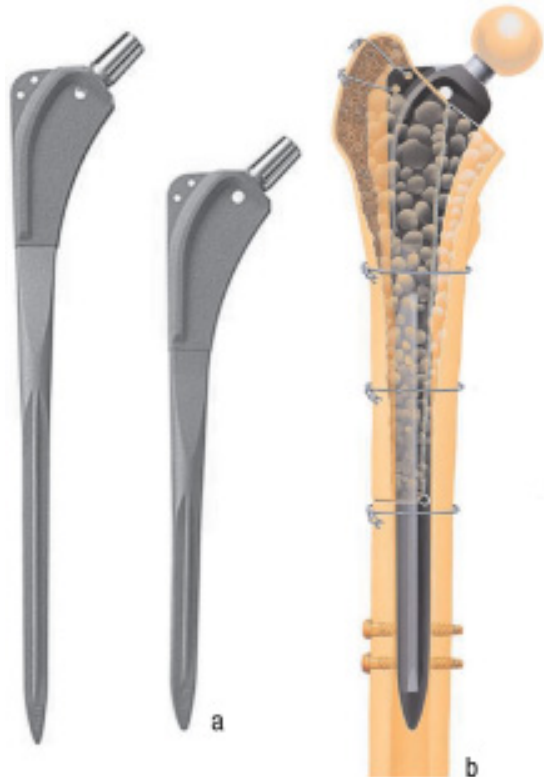

Abb. 6 (a) BiContact-Revisionsschaft ${ }^{\circledR}$ mit unterschiedlichen Längen und Durchmessern, (b) Schema der Press-fit-Verankerung im Bereich des Schaftrohres mit zusätzlicher stabilisierender distaler Verriegelung. Verschluss des Femurrohres bei transfemuralem Zugang mit Drahtcerclagen.

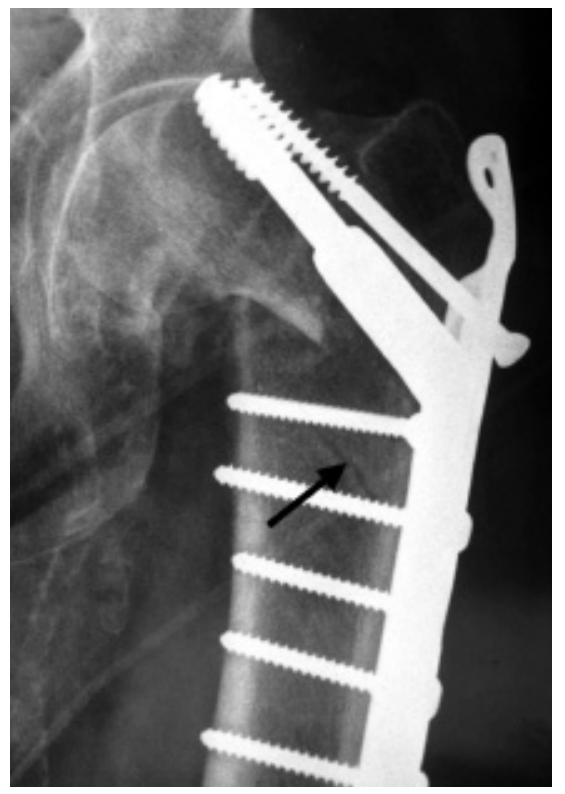

Abb.7a Nach Versorgung einer 31 A 3.1 Fraktur mit einer $\mathrm{DHS}^{\circledR}+$ Trochanterabstützplatte kommt es zu einem „cutting out“ der Kopfschrauben. Pat, weibl. 89 Jahre, schwerwiegende Nebenerkrankungen, deshalb Indikation zur definitiven Versorgung mittels Endoprothese und Trochanterzuggurtung (Pfeil $=$ Frakturverlauf). Alternativ kann man sich eine solche Fraktur als primäre Indikation zur Endoprothese vorstellen, wenn z. B. eine schwerste Coxarthrose vorliegt. lich, da der Hauptverankerungsbereich zwischen dem großen und kleinen Rollhügel durch dessen Einbeziehung in die Fraktur nicht mehr stabil ist. Die Verankerung eines zementierten Prothesenschaftes im Knochenrohr des Femurs ist möglich, dabei ist aber die Verankerung in dem frakturierten Bereich zwischen dem Trochanter major und minor weniger zuverlässig wie bei Intaktheit dieses Areals. Die operative Vorgehensweise entspricht der oben dargestellten, allerdings hat bei diesen Frakturen eine ausreichend gute Refixierung der Trochanteren, insbesondere des Trochanter major, eine sehr große Bedeutung. Es gibt hier unterschiedliche Techniken, in Abb. $\mathbf{3}$ u. 4 sind zwei Möglichkeiten dargestellt. Wird der Trochanter major nicht refixiert, so wird der Patient nicht die erforderliche Weichteilverspannung des operierten Hüftgelenkes zustandebringen, er wird hinken und das Kunstgelenk hat ein großes Risiko zu dislozieren. Die operative Technik der zementierten Schaftimplantation wurde schon ausführlich dargestellt. Im Falle der zusätzlichen Refixierung des Trochanter major sollten vor dem Einzementieren des Schaftes Bohr- löcher im Femurrohr geschaffen werden, durch welche der Cerclagendraht durchgezogen wird. Beim Einzementieren und Aushärten des Zements ist darauf zu achten, dass die Cerclagendrähte korrekt ausgerichtet sind, damit die Zugwirkung optimal wird (Abb. 7c).

Eine Alternative in einer solchen Situation kann die Verwendung eines Revisionsschaftes sein, wenn dieser in der Diaphyse des Femurs zuverlässig verankert werden kann. Seit 10 Jahren besteht in der BG-Klinik Tübingen Erfahrung mit dem BiContact-Revisionsschaft ${ }^{\circledR}$ [6]. Dieser findet seine primäre Verankerung durch einen Press-Sitz und durch eine distale Verriegelung in der Diaphyse des Femurs. Er sollte nur angewendet werden, wenn diese Verankerung auch gut genug gewährleistet ist. Durch diese distale Verankerung ist der Schaft nicht auf eine primär stabile proximale Verankerung „angewiesen“. Vielmehr kann sich in den Monaten nach dem Eingriff das proximale Femurende nach den Prinzipien der Knochenbruchheilung „remodellieren“, d. h. wiederherstellen. Nach sicherer Verankerung des passgerechten

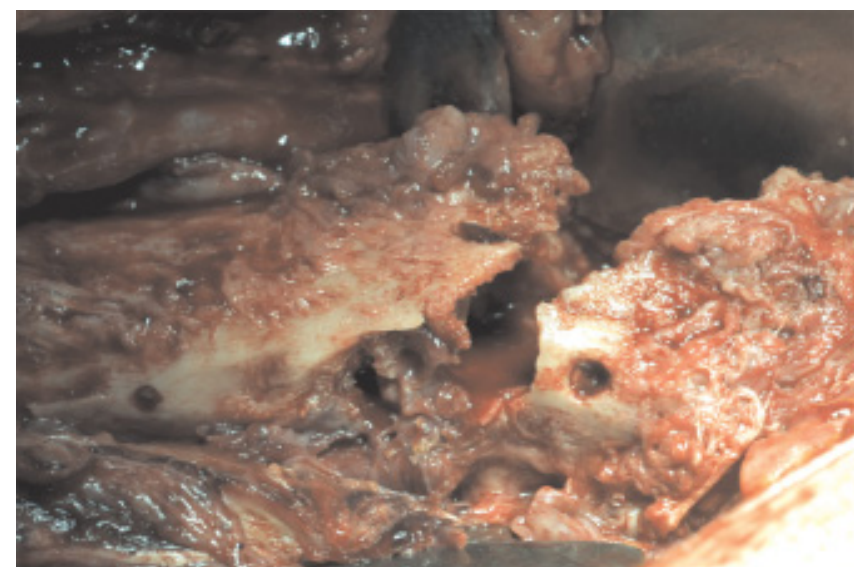

Abb. 7b Coxales Femurende nach Entfernen der $\mathrm{DHS}^{\circledR}$ Das proximale Ende (re. Bildseite) muss mit dem Femurrohr (li. Bildseite) wieder verbunden werden, nachdem in dieses ein zementierter Schaft implantiert wurde.

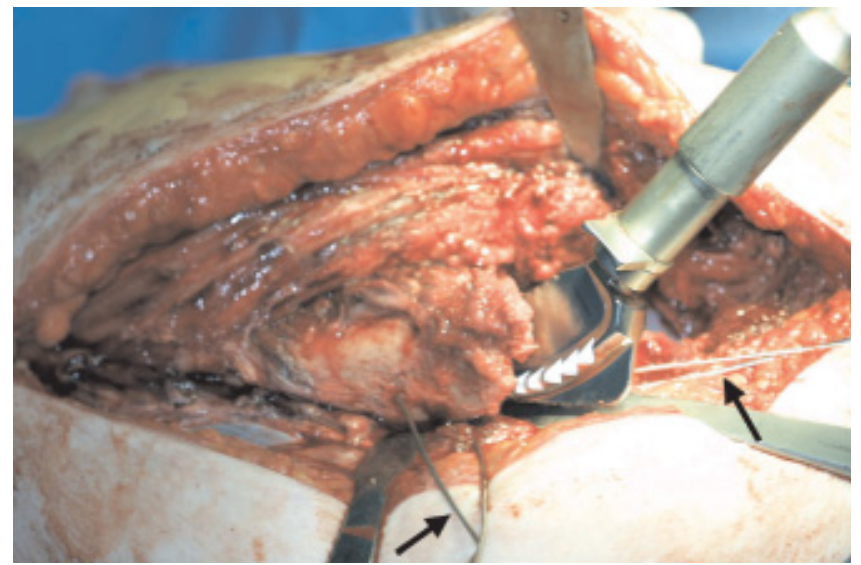

Abb.7c Der Femurschaft wird mittels B-Profiler zur Aufnahme der Schaftprothese vorbereitet. Zuvor wurden 1,5 Drahtcerclagen (Pfeile) am Femurrohr verankert, mit denen das proximale Frakturfragment refixiert wird. 

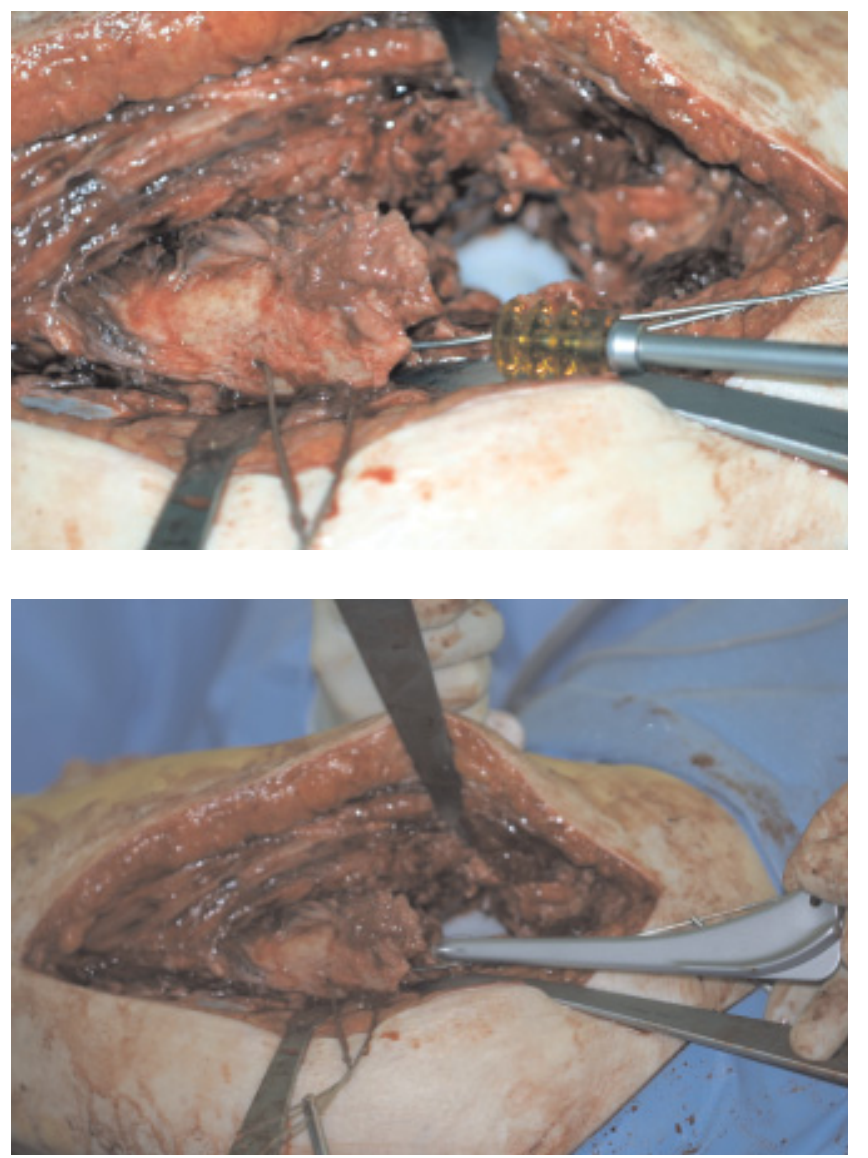

Abb. 7e Einbringen des unbeschichteten BiContact ${ }^{\circledR}$-Schaftes in das mit Zement retrograd aufgefüllte Femur. In der Tiefe ist die mit PMMA fixierte PE-Pfanne zu sehen.

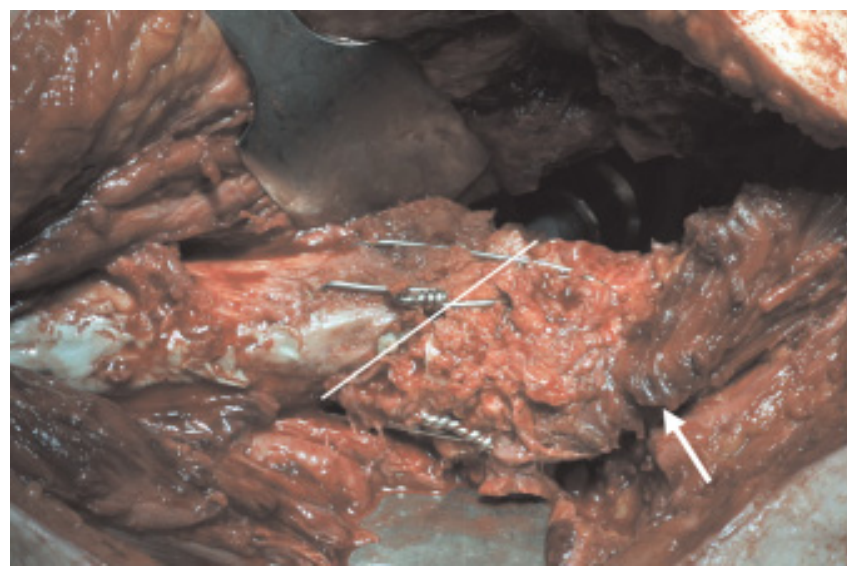

Abb. 7f Situation nach Vervollständigung der komprimierenden Drahtcerclage. An der Andockstelle des Trochanter major an den Schaft darf kein PMMA interponiert sein, dies würde die knöcherne Heilung verhindern. Die am Trochanter ansetzenden Weichteile (Pfeil) sind gut zu erkennen. Weiße Linie $=$ Frakturverlauf.
Schaftes sollten die Fragmente des intertrochanteren Bereiches bestmöglich reponiert und fixiert werden, vorzugsweise durch Cerclagen (Abb.6). Dabei ist aber strikt darauf zu achten, dass die Weichteile so wenig wie möglich von den Knochenfragmenten abgelöst werden, da sonst deren Durchblutung gestört werden kann. Die operative Vorgehensweise entspricht der bisher dargestellten, bei der Schaftpräparation ändern sich jedoch einige Schritte. Zur Vorbereitung des Schaftlagers werden Reibahlen verwendet, die den Sitz des Revisionsschaftes vorbereiten. Dieser wird dann von seiner Größe (Länge und Durchmesser) her so gewählt, dass er einen möglichst optimalen Press-Sitz im Schaftrohr hat und auch ausreichend lang bzw. kurz ist, damit nach der Reposition eine gute Weichteilverspannung mit aufgestecktem Hüftkopf resultiert. Eine zusätzliche distale Verriegelung, wie man sie von der Marknagelung her kennt sorgt für eine weitere wirksame axiale und rotatorische Stabilisierung dieses Implantates (Abb.6 $\mathbf{u}$.8d). Bei dieser Technik handelt es sich um ein „zementfreies“ Verfahren. Bis zur

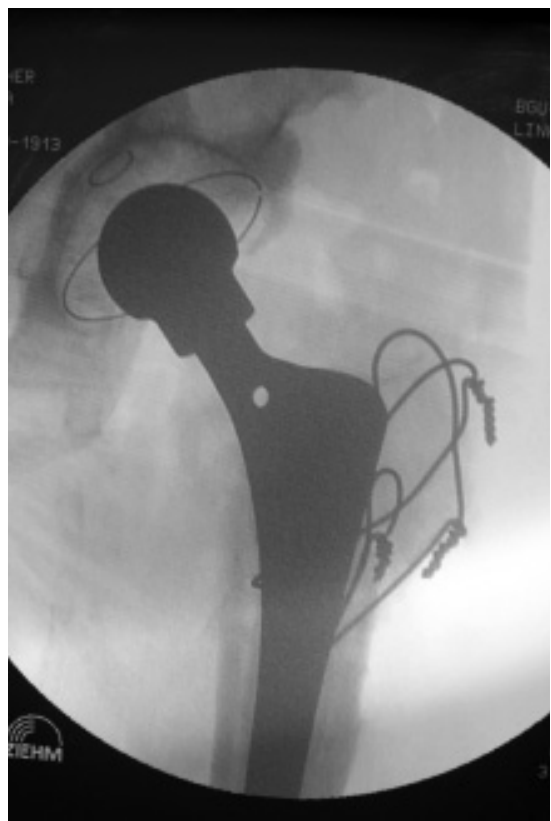

Abb.7g Die intraoperative Rö-Kontrolle zeigt die PE-Pfanne und den Schaft jeweils korrekt positioniert. Der Trochanter major ist mit komprimierenden Cerclagen refixiert. Gute Zementintrusion im Bereich von Pfanne und Schaft. Der mit PMMA aufgefüllte Defekt am oberen Pfannenerker ist durch die ausgewanderte Kopfschraube bedingt.

knöchernen Konsolidierung des Femurrohres ist die operierte Extremität nicht voll belastbar, was für die alten Patienten einen großen Nachteil darstellen kann.

Eine weitere Alternative stellt die Verwendung einer „Tumorprothese“ dar [2] (Abb.2). Deren Indikationsgebiet sollte aber dringend auf durch (fast ausschließlich maligne) Tumoren mit zusätzlicher Frakturierung zerstörte proximale Femurenden beschränkt bleiben. Dafür gibt es zwei Gründe: Ohne Not sollte grundsätzlich keinerlei Knochengewebe geopfert werden, auf bestmöglichen Knochenerhalt ist in der Endoprothetik grundsätzlich immer zu achten. Zweitens setzen am coxalen Femurende wichtige Sehnen und Muskeln an, ohne die ein Hüftgelenk nur schwer stabilisiert werden kann. An den meisten Typen der Tumorprothesen gibt es zwar Verankerungsmöglichkeiten für diese wichtigen stabilisierenden Weichteile, erfahrungsgemäß ist diese Fixierung an einem Implantat qualitativ wesentlich schlechter als über den Knochenkontakt. So wird die Anwendung einer Tumorprothese nur verzweifelten Fällen vorbehalten bleiben, bei denen es um den Extremitätenerhalt geht. 

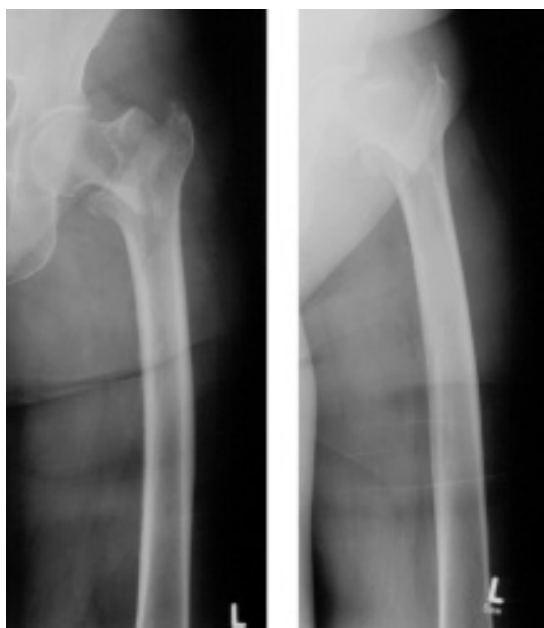

Abb. 8a 78jährige weibl. Pat. mit 31A 1.2Fraktur.
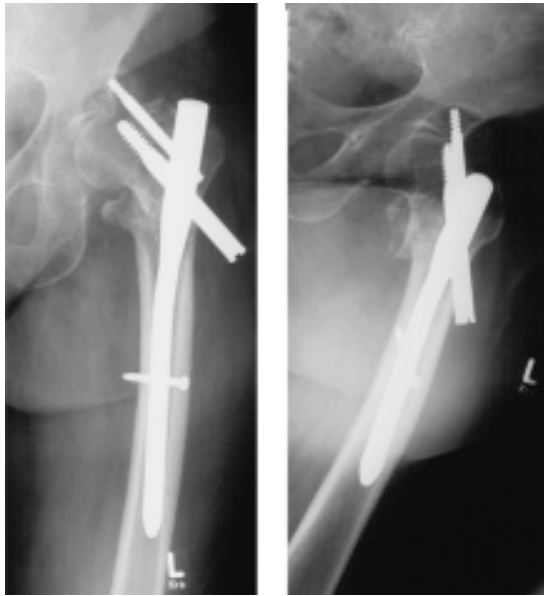

Abb.8c Sekundäre Dislokation dieser Fraktur mit Auswandern der Hüftkopf- und der Gleitschraube.

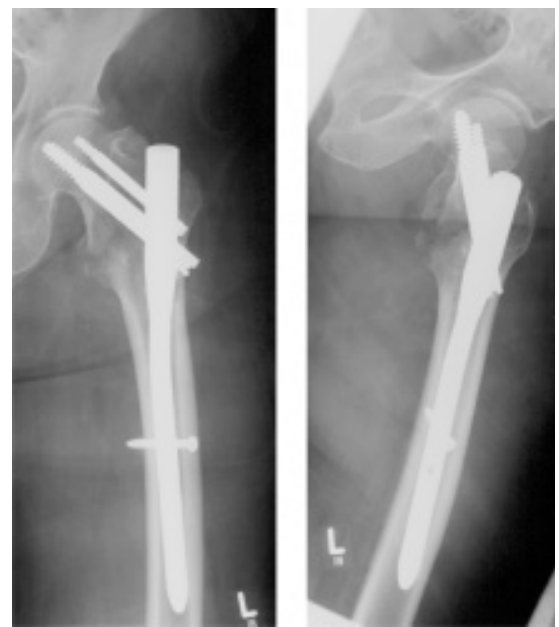

Abb. 8b Versorgung dieser Fraktur mit PFN ${ }^{\circledR}$
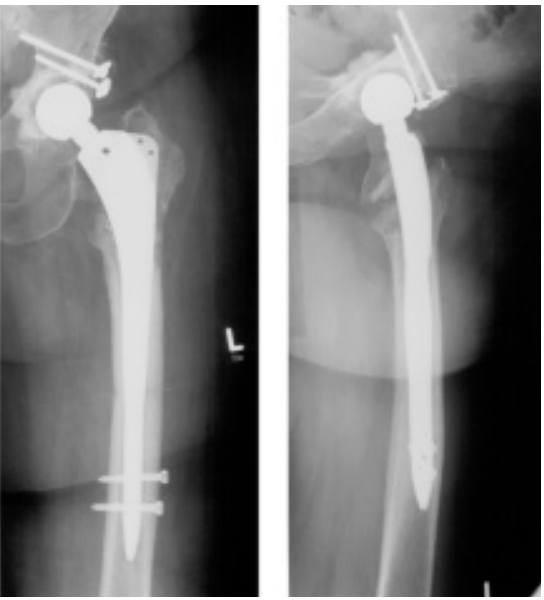

Abb. 8d Nach Ausbau des PFN ${ }^{\circledR}$ erfolgt die Implantation einer Hüftendoprothese: Azetabulär PE-Pfanne mit Hüftpfannenerkerplastik (autogener Knochen) und femural BiContact-Revisionsschaft ${ }^{\circledR}$ mit Press-fit-Verankerung im Femurrohr und zusätzlicher distaler Verriegelung.

da die Verankerungszone erheblich mehr in Mitleidenschaft gezogen ist. Bei Frakturen, die nicht durch eine maligne Erkrankung (meist Metastase, seltener primärer Knochentumor) bedingt sind, ist die Implantation eines Revisionsschaftes (Abb.6 u.8) zu erwägen, wobei der transfemurale Zugang durch die Fraktur schon vorgegeben ist. Wiederum ist auf eine sorgfältige und bestmögliche Refixation des Trochanter major und sofern möglich auch des Trochanter minor zu achten. In den Fällen einer pathologischen Fraktur diesen Typs sollte die Implantation einer Tumorprothese (Abb.9) in Betracht gezogen werden.
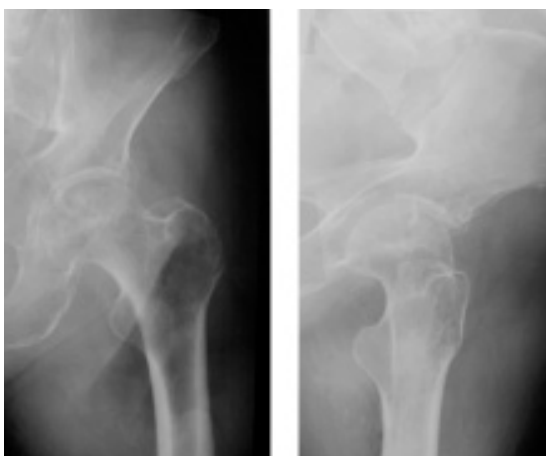

Abb.9a 75jähriger Pat. mit Metastase eines Prostatakarzinoms im coxalen Femurende.

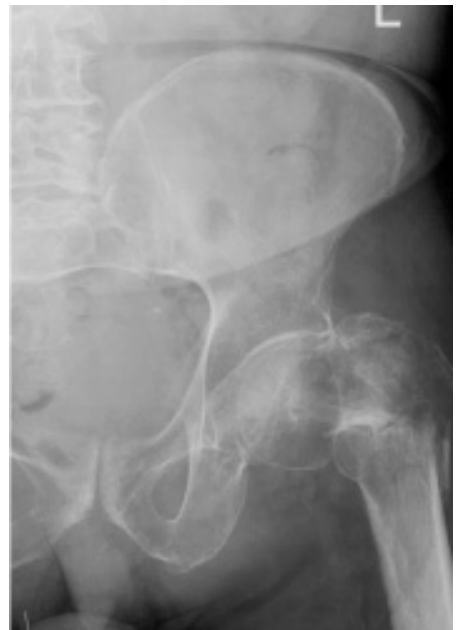

Abb.9b Pertrochantere pathologische Fraktur im Bereich dieser Metastase.
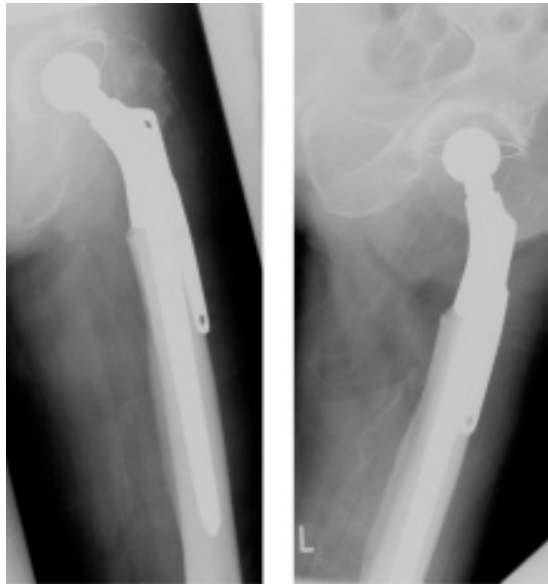

Abb.9c Versorgung dieser Fraktur durch Resektion der Knochenmetastase und Implantation einer zementierten Tumorprothese. Die am Trochanter major inserierenden Weichteile werden an den Verankerungslöchern der Tumorprothese fixiert. auch für diese Frakturengruppe relevant sein. Bei diesen Frakturen wird die Implantation eines Hüftendoprothesenschaftes nochmals eine Stufe schwieriger, 


\section{Schlussfolgerung}

Nachfolgend werden die Ausnahmeindikationen für eine endoprothetische Versorgung der 31 A 1, A 2 und A 3-Frakturen und die in unserer Klinik gebräuchliche entsprechende operative Technik zusammenfassend dargestellt.

a) Vorbestehende versteifende Hüftarthrose bei 31 A 1, A2 oder A3-Frakturen.

In der Zukunft wird eine solche Situation erheblich häufiger auftreten: Ausgehend vom Jahr 1999 ist in Deutschland bei erhöhter Lebenserwartung mit einer Zunahme coxaler Femurfrakturen um bis zu 300\% zu rechnen [7]. Bei den 31 A 1.1 und 31 A 1.2-Frakturen ist die Implantation eines vorzugsweise zementierten Hüftendoprothesenschaftes durchführbar. Liegt eine Fraktur vom Typ 31 A 1.3, 31 A 2 oder 31 A 3 vor, so ist die Verwendung eines Revisionschaftes zu bevorzugen. Auf eine zuverlässige Refixierung des Trochanter major ist dringend zu achten.

b) Kombinierte peritrochantere Frakturen beim alten Menschen

Auch kombinierte Frakturen im trochanteren Bereich (z. B. laterale Schenkelhalsfraktur und subtrochantere Fraktur) werden aufgrund der Veränderung der Alterspyramide mit erheblicher Zunahme alter Menschen zunehmen. Diese Patienten haben häufig zudem eine ausgeprägte Osteoporose. Auch diese Situation erschwert eine stabile osteosynthetische Versorgung erheblich, so dass vermutlich häufiger die Implantation eines Kunstgelenkes erwogen werden muss. In diesen Fällen wird am ehesten die Verwendung eines Revisionsschaftes mit guter Verankerung im Femurrohr angezeigt sein, da die für die Fixation des Standardschaftes wichtige trochantere Region frakturiert ist.

c) Pathologische 31 A1, A2 oder A3-Frakturen

Die Inzidenz pathologischer Frakturen wird zukünftig ansteigen, da aufgrund der heute besseren Therapieoptionen die Überlebenszeit von Patienten mit malignen Erkrankungen steigt und somit immer mehr Patienten das Stadium der Skelettmetastasierung erreichen. Ist das proximale Femurende von einem malignen Prozess betroffen, bietet sich entweder eine Verbundosteosynthese oder die Implantation einer Schaftprothese - meistens einer Tumorprothese - zur Versorgung an. Liegt im Azetabulum ein maligner Knochenprozess vor und hat der Pat eine coxale Femurfraktur, so ist ebenso ein Kunstgelenk am ehesten unter Verwendung eines azetabulären Abstützringes ratsam.

d) Kombination einer instabilen Azetabulumfraktur mit einer proximalen Femurfraktur beim alten Menschen

e) Komplikationen nach Osteosynthesen einer 31 A 1, A 2 oder A 3-Fraktur Die Zahl erforderlicher Osteosynthesen bei dieser Frakturlokalisation wird sich zukünftig vervielfachen. Damit wird auch die Komplikationsrate steigen. Es wird häufiger zu einer Sekundärdislokation der operativ versorgten 31 A 1-3-Frakturen kommen und damit wird sich auch die Notwendigkeit erhöhen, eine Implantation eines Kunstgelenkes als „Ultima Ratio“ zu erwägen.
Zusammengefasst gesagt muss zukünftig vermehrt mit der Notwendigkeit eines primären oder sekundären Einbaus eines Kunstgelenkes bei den 31 A 1-3Frakturen gerechnet werden.

\section{Literatur}

1 Charnley J. Low Friction Arthroplasty of the Hip. Springer, Berlin 1979

2 Jäger M, Löffler L, Kohn D. Tumorprothese des Hüftgelenkes. Z Orthop 1985; 23: $808-814$

3 Kübler M. Die operative Versorgung instabiler trochanterer Frakturen mit dem proximalen Femurnagel. Inauguraldissertation, Eberhard-Karls-Universität Tübingen 2002

4 Müller ME, Nazarian S, Koch P, Schatzger J. The comprehensive classification of fractures of long bones. Springer, Berlin Heidelberg New York 1990

${ }^{5}$ Volkmann R, Eingartner C, Winter E, Weise $\mathrm{K}$, Weller S. Midterm results in 500 titanium alloy Straigth femoral stem prostheses - cemented and uncemented technique. Eur J Orthop Surg Traumatol 1998; 8: 133-139

${ }^{6}$ Volkmann R, Eingartner C, Winter E, Weller S. Hüftprothesenschaftrevision bei ausgeprägter Knochendefektsituation. Unfallchirurgie 1997; 268: 360 - 364

7 Wagner S, Rüter A. Per- und subtrochantäre Femurfrakturen. Unfallchirurg 1999; 102: 206-222

8 Winter E, Weller S, Höntzsch D. Die Pfannenaufbauplastik bei Revisionseingriffen nach operativem Hüftgelenkersatz Op-Journal 1994; 10: $273-279$

Priv.-Doz. Dr. med. Eugen Winter 1. Oberarzt

Prof. Dr. med. Kuno Weise

Ärztlicher Direktor

BG Unfallklinik

Schnarrenbergstr. 95

D-72076 Tübingen 